\title{
Smad4 inhibits cell migration via suppression of JNK activity in human pancreatic carcinoma PANC-1 cells
}

\author{
XUEYING ZHANG, JUNXIA CAO, YUJUN PEI, JIYAN ZHANG and QINGYANG WANG
}

Department of Molecular Immunology, Institute of Basic Medical Sciences, Beijing 100850, P.R. China

Received December 11, 2014; Accepted January 21, 2016

DOI: $10.3892 / \mathrm{ol} .2016 .4427$

\begin{abstract}
Smad4 is a common Smad and is a key downstream regulator of the transforming growth factor- $\beta$ signaling pathway, in which Smad4 often acts as a potent tumor suppressor and functions in a highly context-dependent manner, particularly in pancreatic cancer. However, little is known regarding whether Smad4 regulates other signaling pathways involved in pancreatic cancer. The present study demonstrated that Smad4 downregulates c-Jun N-terminal kinase (JNK) activity using a Smad4 loss-of-function or gain-of-function analysis. Additionally, stable overexpression of Smad4 clearly affected the migration of human pancreatic epithelioid carcinoma PANC-1 cells, but did not affect cell growth. In addition, the present study revealed that upregulation of mitogen-activated protein kinase phosphatase-1 is required for the reduction of JNK activity by Smad4, leading to a decrease in vascular endothelial growth factor expression and inhibiting cell migration. Overall, the present findings indicate that Smad4 may suppress JNK activation and inhibit the tumor characteristics of pancreatic cancer cells.
\end{abstract}

\section{Introduction}

Pancreatic cancer is a devastating disease with an extremely poor prognosis, and patients often do not undergo curative surgery (1). Additionally, effective chemotherapy and radiotherapy treatments for pancreatic cancer are limited (1). The 5-year survival of patients with pancreatic cancer is $0.4-4.0 \%$, and has not significantly improved over the past three decades (2). Elucidating the molecular mechanism of pancreatic cancer may contribute to the early diagnosis and effective therapies for pancreatic cancer. Smad4, also referred to as deleted in pancreatic cancer locus 4 , is localized to chromosome $18 \mathrm{q} 21$, and was originally identified as a signaling mediator of the transforming growth factor- $\beta$

Correspondence to: Mr. Qingyang Wang, Department of Molecular Immunology, Institute of Basic Medical Sciences, 27 Taiping Road, Beijing 100850, P.R. China

E-mail: tansun0532@163.com

Key words: Smad4, JNK, pancreatic cancer, cell migration
(TGF- $\beta$ ) signaling pathway (3). It has been reported that tumor development is induced by a decrease in Smad4 in pancreatic cancer, and mutations of the Smad4 gene predict a poorer prognosis of patients with pancreatic ductal adenocarcinoma and pancreatic cancer (4-6). Furthermore, verbal evidence supports the role of Smad4 as a tumor suppressor gene in pancreatic tumorigenesis (7).

c-Jun N-terminal kinase (JNK) is a member of the mitogen-activated protein kinase (MAPK) family (8). JNK has two ubiquitously expressed isoforms, JNK1 and JNK2, and a tissue-specific isoform JNK3. Each isoform has two different splicing forms, p54 and p46 (9). The activation of JNK is mediated by sequential protein phosphorylation through MAPK kinase (MKK)4 and MKK7, which primarily function as two upstream kinases for JNK activation (10). The inactivation of JNK primarily depends on the dephosphorylation effect of phosphatases, including MAPK phosphatase-1 (MKP-1) (11). Numerous studies have revealed that JNK is pivotal in tumorigenesis by enhancing cell proliferation and migration, and antagonizing apoptosis in digestive system tumors, including hepatocellular carcinoma and pancreatic cancer (12-14). A previous study has demonstrated that JNK is a potential therapeutic target for pancreatic cancer (15). In addition, knocking down JNK or introducing a JNK inhibitor factor results in growth inhibition of human pancreatic carcinoma cells. In a previous study, a mouse model with JNK inhibitor factor inhibits tumor growth and prolongs the survival time of the mice (16).

Various signaling pathways in cells constitute a complex network that interact with each other, which is referred to as cross-talk (17-19). Previous studies have revealed that the Smad signaling pathway downstream of TGF- $\beta$ has complicated interactions with MAPK members, including p38, JNK and extracellular signal-regulated kinases (ERKs) $(19,20)$. Previous studies conducted over the past decade have revealed that the Smad2/3 complex is phosphorylated by JNK and p38 through direct or indirect ways; and this complex subsequently binds to Smad4, thus regulating downstream gene transcription (20). However, little is known regarding whether Smad4 regulates JNK and p38, and whether it affects the occurrence, development and metastasis of tumors. The present study reports that Smad4 suppresses JNK activity, and also inhibits the migration of human pancreatic epithelioid carcinoma PANC-1 cells by upregulating the expression of MKP-1. 


\section{Materials and methods}

Cell culture and transfection. Human embryonic kidney (HEK)-293T, human cervix adenocarcinoma epithelial HeLa and human pancreatic epithelioid carcinoma PANC-1 cells were purchased from the American Type Culture Collection (Manassas, VA, USA). Human pancreatic adenocarcinoma AsPC-1, BxPC-3 and SW850 cells were obtained from Professor Hongyang Wang (National Center for Liver Cancer, Secondary Military Medical University, Shanghai, China). The cells were cultured in Dulbecco's modified Eagle medium (DMEM; Thermo Fisher Scientific, Inc., Waltham, MA, USA) supplemented with $10 \%$ fetal bovine serum (FBS; Lanzhou Bailing Biotechnology Co., Ltd., Lanzhou, China), 100 U/ml penicillin (North China Pharmaceutical Group Co., Ltd., Shijiazhuang, China) and $100 \mathrm{U} / \mathrm{ml}$ streptomycin (North China Pharmaceutical Group Co., Ltd.), and were maintained at $37^{\circ} \mathrm{C}$ with $5 \% \mathrm{CO}_{2}$. Cell transfection was performed using Lipofectamine ${ }^{\circledR} 2000$ (Thermo Fisher Scientific, Inc.), according to the manufacturer's protocol. Stable clones were selected using $800 \mu \mathrm{g} / \mathrm{ml}$ puromycin (Thermo Fisher Scientific, Inc.) for $\sim 2$ months.

Mice. Female BALB/c mice ( $\mathrm{n}=3)$, 6-8 weeks-old, were purchased from Institute of Experimental Animals, Military Medical Sciences Institution (Beijing, China). All mice were maintained at room temperature under specific pathogen-free conditions and exposed to $12 \mathrm{~h} \mathrm{light/dark} \mathrm{cycles.} \mathrm{The} \mathrm{care,}$ use and treatment of mice in the present study was in strict agreement with guidelines in the care and use of Laboratory Animal Manual set out by the Institute of Basic Medical Sciences (Beijing, China). The protocol was approved by the Institute of Basic Medical Sciences. All surgery was performed under sodium pentobarbital anesthesia, and all efforts were made to minimize suffering. Following anesthesia, the mouse thymus was removed, lyzed and subjected to western blot analysis.

Plasmid and small interfering (si) RNA. The HA-Smad4 expression vector was generated by cloning a polymerase chain reaction (PCR) product into the pCDNA3. $1^{+}$vector (Thermo Fisher Scientific, Inc.), which was confirmed by DNA sequencing. The PCR were performed using a MasterCycler ${ }^{\circledR}$ Personal (Eppendorf, Hamburg, Germany). The synthesis of the primers for PCR and the DNA sequencing were performed by Beijing Sino Geno Max Co., Ltd. (Beijing, China). PCR was performed using Taq 2X PCR Mastermix [Tiangen Biotech (Beijing) Co., Ltd., Beijing, China]. The primers for the PCR were as follows: HA-Smad-4, forward 5'-CCCAAGCTTGCCACCATGTACGATGTTCCAGAT TACGCTATGGACAATATGTCTATTACG-3' and reverse 5'-GCTCTAGATACGTCTAAAGGTTGTGGG-3'. Smad4 siRNA (Smad4-1, CGA AUACACCAACAAGUAATT; Smad4-2, AGAUGA AUUGGAUUCUUUATT), MKP-1 siRNA-1 (GCAUAACUGCCUUGAUCAA), MKP-1 siRNA-2 (CCA AUUGUCCCA ACCAUUU) and non-targeting control siRNA (scramble, UUCUCCGAACGU GUCACGUTT) were purchased from Shanghai GenePharma Co., Ltd. (Shanghai, China). siRNAs that target human JNK1 and JNK2 messenger RNA were designed based on the
1,013-1,031 nt (JNK1) and 461-479 nt (JNK2) sequences, relative to the translation start sites, and were purchased from GE Dharmacon (Lafayette, CO, USA) (21). The JNK siRNA sequences were as follows: 5'-CUGACAAGCAGUUAG AUGA-3' for JNK1; 5'-CUAGCA ACAUUGUUGUGAA-3' for JNK2. PCR was performed under the following conditions: Denaturation at $98^{\circ} \mathrm{C}$ for $30 \mathrm{sec}$, followed by 30 cycles of $58^{\circ} \mathrm{C}$ for $1 \mathrm{~min}$, and a final extension step at $72^{\circ} \mathrm{C}$ for $2 \mathrm{~min}$. The PCR products were analyzed by $1.2 \%$ agarose gel (Sigma-Aldrich) electrophoresis and observed under ultraviolet light (UV/White TMW-20 Transilluminator; UVP LLC, Upland, CA, USA).

Western blot analysis. Western blot analysis was performed as previously described (22). Briefly, the cells were washed twice with ice-cold phosphate-buffered saline and were lysed using $20 \mathrm{mM}$ Tris/HCl (pH7.6; Amresco, Inc., Solon, OH, USA), $250 \mathrm{mM} \mathrm{NaCl}, 3 \mathrm{mM}$ EDTA (Sigma-Aldrich, St. Louis, MO, USA), $3 \mathrm{mM}$ ethylene glycol tetraacetic acid (Sigma-Aldrich), 0.5\% NP-40 lysis buffer (Amresco, Inc.), $1 \mathrm{mM}$ dithiothreitol (Sigma-Aldrich), $1 \mathrm{mM}$ p-nitrophenyl phosphate (Sigma-Aldrich), $2 \mathrm{mM} \mathrm{Na}_{3} \mathrm{VO}_{4}$ (Sigma-Aldrich) and $10 \mu \mathrm{g} / \mathrm{ml}$ aprotinin (Sigma-Aldrich). The whole cell extract was centifuged (Fresco 21; Thermo Fisher Scientific, Inc.) at $13,600 \mathrm{x} \mathrm{g}$ for $15 \mathrm{~min}$ at $4^{\circ} \mathrm{C}$, and the supernatants were subjected to $12 \%$ sodium dodecylsulfate-polyacrylamide gel electrophoresis ( $2 \mathrm{~h}$ ). Subsequently, the proteins were transferred to Hybond-P polyvinylidene difluoride membranes (GE Healthcare Life Sciences, Little Chalfont, UK). The membranes were initially incubated with primary antibody at $4^{\circ} \mathrm{C}$ overnight, and then with horseradish peroxidase (HRP)-conjugated secondary antibodies for $1 \mathrm{~h}$ at room temprature. Bound antibody was detected using Amersham ECL Western Blotting Detection kit (catalog no. RPN2106; GE Healthcare Life Sciences) and X-ray film (Kodak, Rochester, NY, USA). The following primary antibodies were used in a dilution of 1:1,000: Rabbit polyclonal anti-human phosphorylated (p-)JNK (catalog no. 9251; Cell Signaling Technology, Inc., Danvers, MA, USA), rabbit monoclonal anti-human p-p38 MAPK (catalog no. 9215; Cell Signaling Technology, Inc.), mouse monoclonal anti-human JNK1 (catalog no. 51-1570GR; BD Pharmingen ${ }^{\mathrm{TM}}$; BD Biosciences, Franklin Lakes, NJ, USA), rabbit monoclonal anti-human JNK2 (catalog no., 2037-1; Epitomics, Burlingame, CA, USA), rabbit polyclonal anti-human p38 (catalog no. sc-535; Santa Cruz Biotechnology, Inc., Dallas, TX, USA), rabbit polyclonal anti-hemagglutinin (HA)-probe (catalog no. sc-805; Santa Cruz Biotechnology, Inc.), rabbit polyclonal anti-human Smad4 (catalog no. 9515; Cell Signaling Technology, Inc.,), rabbit polyclonal anti-human MKP-1 (catalog no. sc-1199; Santa Cruz Biotechnology, Inc.), monoclonal mouse anti-human vascular endothelial growth factor (VEGF; catalog no. MAB293; R\&D Systems, Inc., Minneapolis, MN, USA) and mouse monoclonal anti-human $\beta$-actin (catalog no. sc-47778; Santa Cruz Biotechnology, Inc.). A polyclonal goat anti-rabbit or goat anti-mouse HRP-conjugated secondary antibody (catalog no. ZB-2301 and ZB-2305, respectively; both dilution 1:5000; Zhongshan Golden Bridge Biotechnology Co., Ltd., Beijing, China) were used at a dilution of 1:5,000. Densitometric analysis was 
A
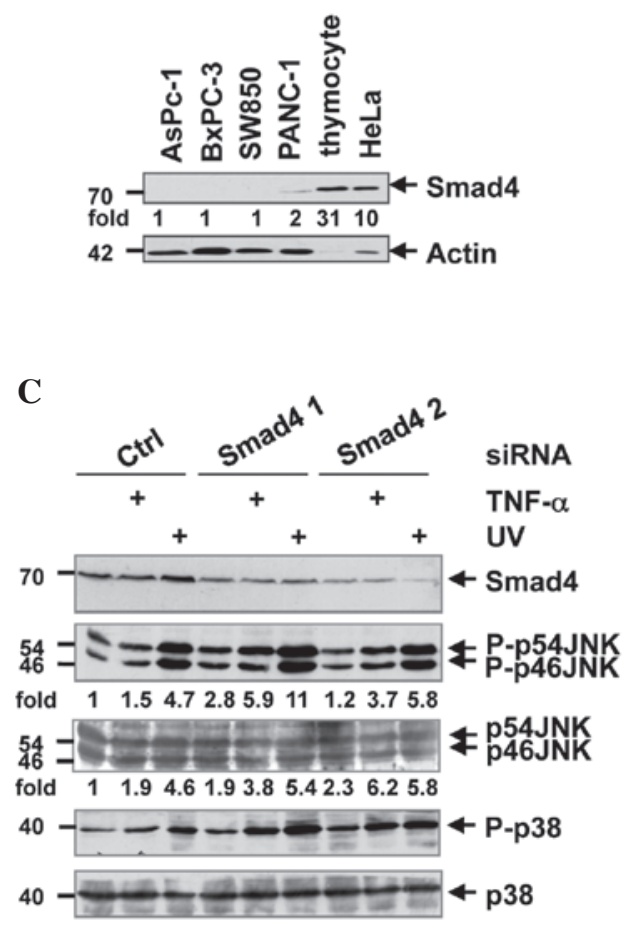

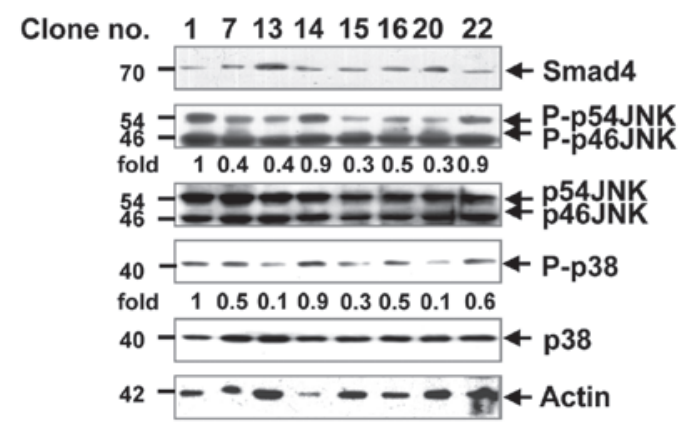

D

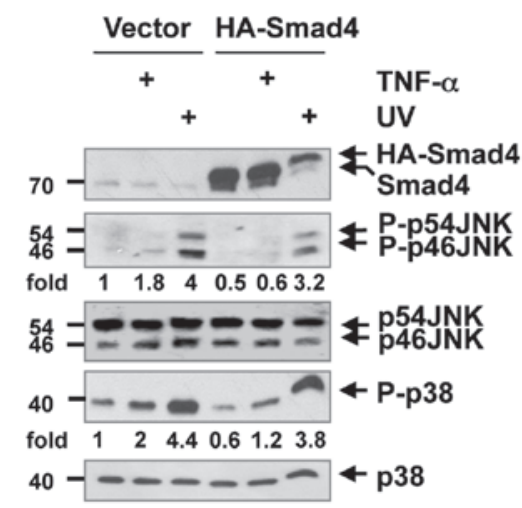

Figure 1. Smad4 suppressed JNK activity in human pancreatic epithelioid carcinoma PANC-1 and HEK-293T cells. (A) Human pancreatic adenocarcinoma AsPC-1, BxPC-3, SW850 and PANC-1 cells, human epithelial carcinoma HeLa cells and mouse thymocyte tissue were lysed with a lysis buffer, and the lysates were subjected to western blot analysis for the detection of Smad4 and actin. DR are shown for Smad4 and normalized with actin. (B) PANC-1 single clones that stably expressed Smad4 were lysed and subjected to western blot analysis with antibodies against Smad4, p-JNK, JNK, p-p38, p38 and actin. (C) HEK-293T cells were transfected with control or Smad4 siRNA. Following incubation for $72 \mathrm{~h}$, the cells were stimulated with TNF- $\alpha$ or UV light for 15 min, and lysed on ice. The lysates were subjected to western blot analysis with antibodies against Smad4, p-JNK, JNK, p-p38 and p38. (D) HEK-293T cells were transfected with control vector or and hemagglutinin-Smad4 plasmid. Following TNF- $\alpha$ or UV light stimulation $24 \mathrm{~h}$ later, the cells were lysed, and the cell lysates were subjected to western blot analysis with antibodies against Smad4, p-JNK, JNK, p-p38 and p38. (B-D) DR are shown for p-JNK and normalized with JNK; DR are shown for p-p38 and normalized with p38. HEK, human embryonic kidney; p-, phosphorylated; JNK, c-Jun N-terminal kinase; siRNA; small interfering RNA; TNF, tumor necrosis factor; UV, ultraviolet; HA, hemagglutinin probe; Ctrl, control; DR, densitometric readings.

performed using Gel-Pro Analyzer 4.0 (Media Cybernetics, Inc., Rockville, MD, USA).

Cell proliferation and migration assay. Cells were seeded into a 24-well plate at a density of $2 \times 10^{4}$ cells/well for $12 \mathrm{~h}$. Cells were then incubated with $20 \mu \mathrm{M}$ SP600125 (JNK inhibitor; Sigma-Aldrich) or $20 \mu \mathrm{M}$ dimethyl sulfoxide. The viable cells were counted daily with a trypan blue stain (Yeasen Corporation, Shanghai, China). A migration assay was performed using a Transwell chamber (diameter, $6.5 \mathrm{mM}$; pore size, $8 \mu \mathrm{M}$; polycarbonate membrane; Corning Incorporated, Corning, NY, USA). In total, $3 \times 10^{4}$ PANC-1 cells in $0.1 \mathrm{ml}$ serum-free DMEM were placed in the upper chamber, while the lower chamber was loaded with $0.5 \mathrm{ml} 10 \%$ FBS-DMEM as a chemoattractant. Following incubation at $37^{\circ} \mathrm{C}$ and $5 \% \mathrm{CO}_{2}$ for $6 \mathrm{~h}$, the non-invading cells in the inserts were removed with cotton swabs. The migrated cells on the lower surface were stained with sulforhodamine B (Sigma-Aldrich) and counted under a microscope (ECLIPSE TS100; Nikon Corporation, Tokyo, Japan) in five randomly selected fields at a magnification of $\mathrm{x} 400$.

Statistical analysis. Data are expressed as the mean \pm standard deviation. The Student's t-test was used to compare the differ- ences between groups. All statistical analysis was performed using SPSS 13.0 software (SPSS, Inc., Chicago, IL, USA). $\mathrm{P}<0.05$ was considered to indicate a statistically significant difference.

\section{Results}

Smad4 suppressed JNK activity in PANC-1 cells and HEK-293T cells. To investigate whether Smad4 affects JNK activity in human pancreatic cancer cells, the present study first analyzed the effects of Smad4 using a gain-of-function analysis in PANC-1 cells (Fig. 1A). In addition, single clone PANC-1 cells that express stable levels of exogenous Smad4 were established. Clones 7, 13, 14, 15 16, 20 and 22 were screened as exogenous Smad4 stably expressing clones, while clone 1 was referred to as a control clone transfected with a pCDNA3.1(+) empty vector. Western blot analysis revealed that these single clone cells exhibited a reduced JNK phosphorylation compared to the control clone, which was associated with Smad4 expression, particularly in clone no. 20 (Fig. 1B). Additionally, the present study investigated whether JNK phosphorylation was suppressed by Smad4 in non-pancreatic cells. A transient knockdown of Smad4 using siRNA partially elevated basal and tumor necrosis factor 
A

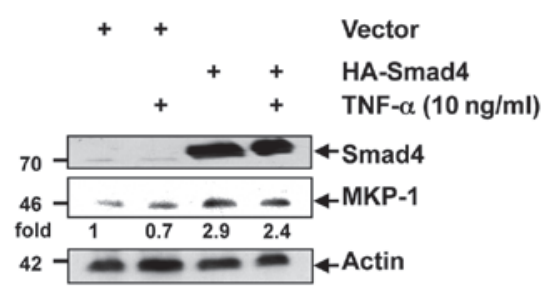

C

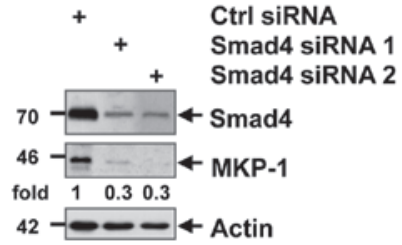

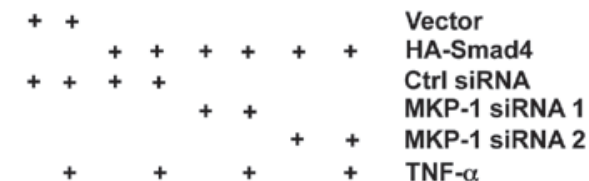

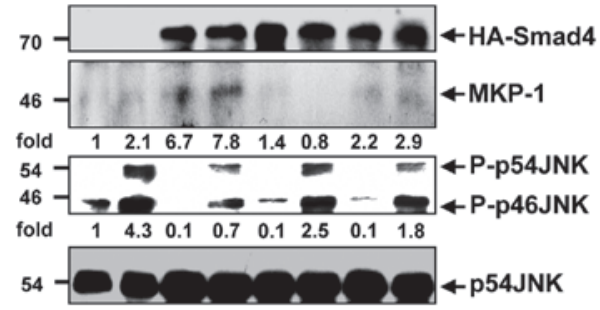

Figure 2. Smad4 upregulated the expression of MKP-1. (A) HEK-293T cells were transfected with control vector or HA-Smad4 plasmid. Following 24 h, the cells were stimulated with TNF- $\alpha$, and the protein levels of Smad4, MKP-1 and actin were analyzed using western blot analysis. (B) HEK-293T cells were transfected with control or Smad4 siRNA, and $72 \mathrm{~h}$ later, the expression levels of Smad4, MKP-1 and actin were analyzed using western blot analysis. DR are shown for MKP-1 and normalized with actin. (C) HEK-293T cells were co-transfected with control vector or HA-Smad4 plasmid and control or MKP-1 siRNA. Following 15 min of TNF- $\alpha$ stimulation, the cells were lysed, and the expression levels of Smad4, MKP-1, JNK and phosphorylated JNK were analyzed using western blot analysis. DR are shown for p-JNK and normalized with JNK; densitometric readings are shown for MKP-1 and normalized with JNK. MKP-1, mitogen-activated protein kinase phosphatase-1; HEK, human epithelial kidney; TNF, tumor necrosis factor; p-, phosphorylated; JNK, c-Jun N-terminal kinase; siRNA; small interfering RNA; HA, hemagglutinin; Ctrl, control; DR, densitometric readings.

A

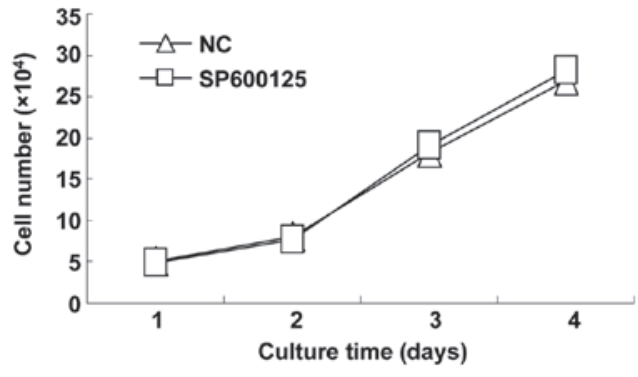

C

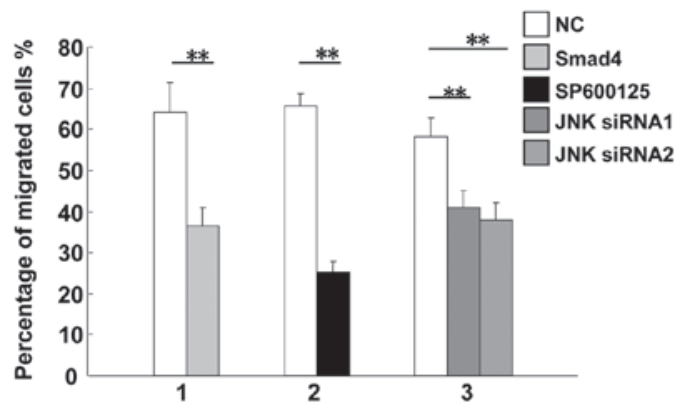

B

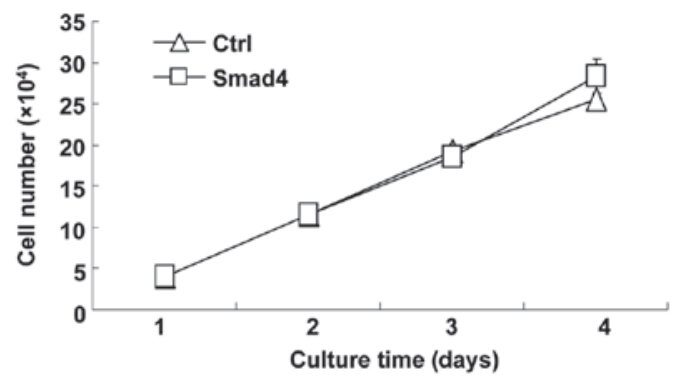

D

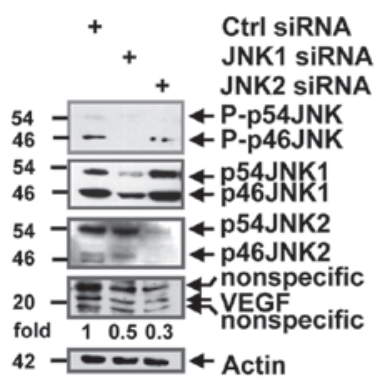

Figure 3. Smad4 suppressed cell migration, but not proliferation. (A) Human pancreatic epithelioid carcinoma PANC-1 cells were seeded into 24 -well plates at a density of $2 \times 10^{4}$ cells/well, and treated with DMSO or SP600125, a JNK inhibitor. The cells were counted daily between days 1 and 4 . (B) PANC-1 single clones that stably expressed the control vector or Smad4 were seeded into 24 -well plates at a density of $2 \times 10^{4}$ cells/well, and counted daily between days 1 and 4. (C) PANC-1 cells subjected to various treatments were analyzed by Transwell assay. The migrated cells on the lower surface of the membrane were stained with sulforhodamine B, and counted under a microscope in five randomly selected fields at a magnification of x400. Group 1, cells transfected with control vector or hemagglutinin-Smad4 plasmid; group 2, cells treated with DMSO or the JNK inhibitor SP600125; group 3, cells transfected with control or JNK siRNA. (D) PANC-1 cells were transfected with control, JNK1 or JNK2 siRNA. The cells were lysed following $72 \mathrm{~h}$, and the lysates were subjected to western blot analysis with antibodies against phosphorylated JNK, JNK1, JNK2, vascular endothelial growth factor and actin. DR are shown for VEGF and normalized with actin. ${ }^{* *} \mathrm{P}<0.01$ vs. NC. JNK, c-Jun N-terminal kinase; siRNA; small interfering RNA; DMSO, dimethyl sulfoxide; p-, phosphorylated; VEGF, vascular endothelial growth factor; $\mathrm{NC}$, negative control; Ctrl, control; DR, densitometric readings.

(TNF)- $\alpha$ (10 ng/mL; R\&D Systems, Inc.) or 302 nM UV light (CL-1000M Midrange Ultraviolet Crosslinker; UVP, Inc., Upland, CA, USA)-induced JNK and p38 phosphorylation compared to control siRNA in HEK-293T cells (Fig. 1C). Similarly, overexpression of Smad4 in HEK-293T cells attenuated the phosphorylation of JNK and p38 (Fig. 1D). 
These results suggest that Smad4 suppressed JNK activity in various cell types.

Smad4 suppressed JNK activity by upregulating MKP-1. MKP-1 is a nuclear-localized phosphatase that dephosphorylates MAPK ERK, p38 and JNK (23). To investigate the potential role of MKP-1 in the association between Smad4 and JNK, HA-Smad4 and Smad4 siRNA were transfected into PANC-1 cells. Western blot analysis demonstrated that MKP-1 protein was upregulated with Smad4 overexpression, but downregulated in Smad4 knockdown cells (Fig. 2A and B). To additionally examine the role of MKP-1 in this biological process, PANC-1 cells were transfected with the HA-Smad4 vector, followed by silencing of MKP-1 using siRNA, and stimulation with TNF- $\alpha$. As expected, the suppression of JNK phosphorylation by Smad4 was partially reversed under the conditions of MKP-1 knockdown (Fig. 2C). Therefore, MKP-1 upregulation was pivotal in Smad4-regulated suppression of JNK phosphorylation.

Smad4 suppressed migration, but not proliferation, via JNK activity in PANC-1 cells. JNK activity has been reported to contribute to oncogenicity by promoting tumor cell proliferation and migration (24). Therefore, it is important to investigate whether Smad4 affects the oncogenicity of PANC-1 cells via JNK. Notably, a PANC-1 single clone that stably expressed HA-Smad4 exhibited no differences, compared with the control vector (Fig. 3A). The JNK inhibitor also exhibited no effects on PANC-1 cell proliferation (Fig. 3B). However, in the migration analysis, the PANC-1 cells that stably overexpressed Smad4 exhibited a weakened ability for migration. Knockdown of JNK and the use of the JNK inhibitor demonstrated a similar ability to attenuate the migration of the PANC-1 cells (Fig. 3C; $\mathrm{P}<0.01$ ). VEGF is a key factor in tumor cell migration (25). Therefore, JNK siRNAs were used in the present study to examine the association between JNK activity and migration in PANC-1 cells. Silencing of JNK1 and JNK2 using siRNA inhibited VEGF expression in PANC-1 cells (Fig. 3D). These results collectively suggest that Smad4 regulates cell migration by suppressing JNK activity and JNK-dependent VEGF expression.

\section{Discussion}

Pancreatic cancer is a devastating disease with an extremely poor prognosis and a $0.4-4 \%$ survival rate (2). Elucidating the molecular mechanisms of pancreatic cancer may contribute to the early diagnosis and effective therapies for patients with pancreatic cancer. It has been demonstrated that Smad4 deletions are associated with pancreatic cancer metastasis, and Smad4 gene mutations are associated with poor prognosis in pancreatic cancer $(1,2)$. Previous studies have established that Smad4 may interact with other signaling pathways, including Notch and MAPK, and function independently of TGF- $\beta$ (20). Despite clear evidence of the association between Smad4 and pancreatic cancer $(1,4-6)$, the mechanism of the cross-talk between Smad4 and other signaling pathways that affect the development of pancreatic cancer remains unclear.

JNK activity is reported to be pivotal in tumor development, particularly in gastrointestinal tumors, including hepatocellular carcinoma (12), colon cancer (26) and pancreatic cancer (27).
In human colorectal cancer, JNK phosphorylates Smad2/3, which results in normal colorectal epithelial cells transforming to invasive adenocarcinoma (28). However, whether and how Smad4 regulates JNK activity in pancreatic cancer is unknown. The present study demonstrated that Smad4 suppresses JNK activity by elevating MKP1 expression, resulting in reduced VEGF expression and inhibited cell migration. For additional confirmation of these results, the JNK inhibitor SP600125 and JNK siRNA were used in the present study. The present results revealed that the inhibition of JNK by siRNA or SP600125 impaired cell migration and caused overexpression of Smad4 in PANC-1 cells. Overall, the present results suggest that Smad4 acts as a tumor suppressor not only through the TGF- $\beta$ pathway, but also through the JNK/MAPK pathway.

It is of interest that Smad4 inhibits JNK activity not only in malignant cells, but also in normal cells $(29,30)$. Western blot analysis performed in the present study indicated that Smad4 also suppressed JNK activity in HEK-293T cells, suggesting that the Smad4-mediated suppression of JNK may be a universal event. Furthermore, in isolated murine T cells, JNK phosphorylation was inversely associated with Smad4 protein expression (data not shown). Previous studies have indicated that constitutive JNK activity may promote the malignancy of B and T cells $(21,31)$, while mice with a Smad4 deletion were observed to spontaneously develop gastrointestinal cancer $(32,33)$. The possibility that a Smad4 deletion may initiate pancreatic cancer in humans is unclear. The data presented in the current study associated a decrease in Smad4 expression with increased migration of PANC-1 cells, which was accompanied by enhanced JNK activity due to the lack of Smad4. Therefore, the present results suggest that a Smad4 deletion may cause the development of pancreatic cancer, which may be accelerated due to increased JNK activity.

\section{Acknowledgements}

This study was supported by the Basic Research for Application Foundation of General Logistic Department (grant no. 13QNP149).

\section{References}

1. Lepage C, Capocaccia R, Hackl M, Lemmens V, Molina E, Pierannunzio D, Sant M, Trama A and Faivre J: Survival in patients with primary liver cancer, gallbladder and extrahepatic biliary tract cancer and pancreatic cancer in Europe 1999-2007: Results of EUROCARE-5. Eur J Cancer pii: S0959-8049(15)00714-5, 2015.

2. Hruban RH and Adsay NV: Molecular classification of neoplasms of the pancreas. Hum Pathol 40: 612-623, 2009.

3. Hahn SA, Schutte M, Hoque AT, Moskaluk CA, da Costa LT, Rozenblum E, Weinstein CL, Fischer A, Yeo CJ, Hruban RH and Kern SE: DPC4, a candidate tumor suppressor gene at human chromosome 18q21.1. Science 271: 350-353, 1996.

4. Oshima M, Okano K, Muraki S, Haba R, Maeba T, Suzuki Y and Yachida S: Immunohistochemically detected expression of 3 major genes (CDKN2A/p16, TP53, and SMAD4/DPC4) strongly predicts survival in patients with resectable pancreatic cancer. Ann Surg 258: 336-346, 2013.

5. Singh P, Srinivasan R and Wig JD: SMAD4 genetic alterations predict a worse prognosis in patients with pancreatic ductal adenocarcinoma. Pancreas 41: 541-546, 2012.

6. Blackford A, Serrano OK, Wolfgang CL, Parmigiani G, Jones S, Zhang X, Parsons DW, Lin JC, Leary RJ, Eshleman JR, et al: SMAD4 gene mutations are associated with poor prognosis in pancreatic cancer. Clin Cancer Res 15: 4674-4679, 2009. 
7. McCarthy DM, Hruban RH, Argani P, Howe JR, Conlon KC, Brennan MF, Zahurak M, Wilentz RE, Cameron JL, Yeo CJ, et al: Role of the DPC4 tumor suppressor gene in adenocarcinoma of the ampulla of Vater: analysis of 140 cases. Mod Pathol 16: $272-278,2003$

8. Johnson GL and Lapadat R: Mitogen-activated protein kinase pathways mediated by ERK, JNK, and p38 protein kinases. Science 298: 1911-1912, 2002.

9. Wang J, Tang R, Lv M, Wang Q, Zhang X, Guo Y, Chang H, Qiao C, Xiao H, Li X, et al: Defective anchoring of JNK1 in the cytoplasm by MKK7 in Jurkat cells is associated with resistance to Fas-mediated apoptosis. Mol Biol Cell 22: 117-127, 2011.

10. Takekawa M, Tatebayashi K and Saito H: Conserved docking site is essential for activation of mammalian MAP kinase kinases by specific MAP kinase kinase kinases. Mol Cell 18: 295-306, 2005.

11. Liu Y, Gorospe M, Yang C and Holbrook NJ: Role of mitogen-activated protein kinase phosphatase during the cellular response to genotoxic stress. Inhibition of c-Jun N-terminal kinase activity and AP-1-dependent gene activation. J Biol Chem 270: 8377-8380, 1995.

12. Guo Y, Wang W, Wang J, Feng J, Wang Q, Jin J, Lv M, Li X, Li Y, Ma Y, et al: Receptor for activated C kinase 1 promotes hepatocellular carcinoma growth by enhancing mitogen-activated protein kinase kinase 7 activity. Hepatology 57: 140-151, 2013.

13. Sakurai T, Maeda S, Chang L and Karin M: Loss of hepatic NF-kappa B activity enhances chemical hepatocarcinogenesis through sustained c-Jun N-terminal kinase 1 activation. Proc Natl Acad Sci USA 103: 10544-10551, 2006.

14. Kuntzen C, Sonuc N, De Toni EN, Opelz C, Mucha SR, Gerbes AL and Eichhorst ST: Inhibition of c-Jun-N-terminal-kinase sensitizes tumor cells to CD95-induced apoptosis and induces G2/M cell cycle arrest. Cancer Res 65: 6780-6788, 2005.

15. Pearson G, Robinson F, Beers Gibson T, Xu BE, Karandikar M, Berman K and Cobb MH: Mitogen-activated protein (MAP) kinase pathways: Regulation and physiological functions. Endocr Rev 22: 153-183, 2001.

16. Takahashi R, Hirata Y, Sakitani K, Nakata W, Kinoshita H, Hayakawa Y, Nakagawa H, Sakamoto K, Hikiba Y, Ijichi H, et al: Therapeutic effect of c-Jun N-terminal kinase inhibition on pancreatic cancer. Cancer Sci 104: 337-344, 2013.

17. Thornley JA, Trask HW, Ringelberg CS, Ridley CJ, Wang S, Sal-Lari RC, Moore JH, Korc M and Tomlinson CR: SMAD4-dependent polysome RNA recruitment in human pancreatic cancer cells. Mol Carcinog 51: 771-782, 2012.

18. Derynck R and Zhang YE: Smad-dependent and Smad-independent pathways in TGF-beta family signalling. Nature 425: 577-584, 2003.

19. Hayashida T, Decaestecker M and Schnaper HW: Cross-talk between ERK MAP kinase and Smad signaling pathways enhances TGF-beta-dependent responses in human mesangial cells. FASEB J 17: 1576-1578, 2003.

20. He S, Liu X, Yang Y, Huang W, Xu S, Yang S, Zhang X and Roberts MS: Mechanisms of transforming growth factor beta(1)/Smad mediated by mitogen-activated protein kinase pathways in keloid fibroblasts. Br J Dermatol 162: 538-546, 2010
21. Cui J, Wang Q, Wang J, Lv M, Zhu N, Li Y, Feng J, Shen B and Zhang J: Basal c-Jun NH2-terminal protein kinase activity is essential for survival and proliferation of T-cell acute lymphoblastic leukemia cells. Mol Cancer Ther 8: 3214-3222, 2009.

22. Zhang J, Wang Q, Zhu N, Yu M, Shen B, Xiang J and Lin A: Cyclic AMP inhibits JNK activation by CREB-mediated induction of c-FLIP(L) and MKP-1, thereby antagonizing UV-induced apoptosis. Cell Death Differ 15: 1654-1662, 2008.

23. Chi H, Barry SP, Roth RJ, Wu JJ, Jones EA, Bennett AM and Flavell RA: Dynamic regulation of pro- and anti-inflammatory cytokines by MAPK phosphatase 1 (MKP-1) in innate immune responses. Proc Natl Acad Sci USA 103: 2274-2279, 2006.

24. Wagner EF and Nebreda AR. Signal integration by JNK and p38 MAPK pathways in cancer development. Nat Rev Cancer 9: 537-549, 2009

25. Kinney CM, Chandrasekharan UM, Mavrakis L and DiCorleto PE: VEGF and thrombin induce MKP-1 through distinct signaling pathways: Role for MKP-1 in endothelial cell migration. Am J Physiol Cell Physiol 294: C241-C250, 2008.

26. Kwon GT, Cho HJ, Chung WY, Park KK, Moon A and Park JH: Isoliquiritigenin inhibits migration and invasion of prostate cancer cells: Possible mediation by decreased JNK/AP-1 signaling. J Nutr Biochem 20: 663-676, 2009.

27. Li M, Feurino LW, Li F, Wang H, Zhai Q, Fisher WE, Chen C and Yao Q: Thymosinalphal stimulates cell proliferation by activating ERK1/2, JNK, and increasing cytokine secretion in human pancreatic cancer cells. Cancer Lett 248: 58-67, 2007.

28. Yamagata H, Matsuzaki K, Mori S, Yoshida K, Tahashi Y, Furukawa F, Sekimoto G, Watanabe T, Uemura Y, Sakaida N, et al: Acceleration of Smad2 and Smad3 phosphorylation via c-Jun $\mathrm{NH}(2)$-terminal kinase during human colorectal carcinogenesis. Cancer Res 65: 157-165, 2005.

29. Itatani Y, Kawada K, Fujishita T, Kakizaki F, Hirai H, Matsumoto $\mathrm{T}$, Iwamoto $\mathrm{M}$, Inamoto $\mathrm{S}$, Hatano $\mathrm{E}$, Hasegawa S, et al: Loss of SMAD4 from colorectal cancer cells promotes CCL15 expression to recruit CCR $1^{+}$myeloid cells and facilitate liver metastasis. Gastroenterology 145: 1064-1075, 2013.

30. Aitchison AA, Veerakumarasivam A, Vias M, Kumar R, Hamdy FC, Neal DE and Mills IG: Promoter methylation correlates with reduced Smad4 expression in advanced prostate cancer. Prostate 68: 661-674, 2008.

31. Gururajan M, Chui R, Karuppannan AK, Ke J, Jennings CD and Bondada S: c-Jun N-terminal kinase (JNK) is required for survival and proliferation of B-lymphoma cells. Blood 106 1382-1391, 2005.

32. Hahn JN, Falck VG and Jirik FR: Smad4 deficiency in T cells leads to the Th17-associated development of premalignant gastroduodenal lesions in mice. J Clin Invest 121: 4030-4042, 2011.

33. Chen YW, Hsiao PJ, Weng CC, Kuo KK, Kuo TL, Wu DC, Hung WC and Cheng KH: SMAD4 loss triggers the phenotypic changes of pancreatic ductal adenocarcinoma cells. BMC Cancer 14: 181, 2014. 